\title{
Sinonasal Undifferentiated Carcinoma and Esthesioneuroblastoma Recurring as Nonintestinal Adenocarcinoma
}

\author{
Stephen Y. Kang, MD; Jonathan B. McHugh, MD; Stephen E. Sullivan, MD; \\ Lawrence J. Marentette, MD; Erin L. McKean, MD
}

\begin{abstract}
Numerous distinct neoplasms are encountered at the anterior cranial base. Management of these primary tumors and their locoregional recurrences are dictated by the histopathologic diagnosis. We present two unusual cases of extra-axial anterior cranial base malignancies with locoregional recurrence where the recurrent tumor encountered was of a distinct histopathologic type. While rare, this report highlights the possibility of encountering a distinct tumor type in the posttreatment surveillance of patients with anterior cranial base malignancies.
\end{abstract}

Key Words: Esthesioneuroblastoma, sinonasal undifferentiated carcinoma, SNUC, adenocarcinoma, skull base.

Laryngoscope, 123:1121-1124, 2013

\section{INTRODUCTION}

The management of extra-axial malignancies involving the anterior cranial base is largely predicated on the histopathology of the tumor. Numerous distinct neoplasms are encountered in this region, and accurate histopathological diagnosis determines the appropriate treatment plan for these patients. Similarly, the initial management of locoregional recurrence of these tumors is often guided by the known biology of the original tumor. We present two cases of anterior cranial base malignancies with suspected locoregional recurrence. However, in both cases, the recurrent tumor encountered was of a distinct histopathologic type.

\section{CASE REPORT}

\section{Patient 1}

A 39-year-old man presented with nasal obstruction and recurrent epistaxis. Magnetic resonance imaging (MRI) of the skull base showed a mass centered in the right posterior ethmoid that extended into the superior nasal cavity, penetrating the cribriform plate into the anterior

From the Department of Otolaryngology (s.Y.K, S.E.S., L.J.M., E.L.M.), Department of Pathology (J.B.M.), Department of Neurosurgery (s.E.s., L.J.M., E.L.M.), University of Michigan Health System, Ann Arbor, Michigan, U.S.A.

Editor's Note: This Manuscript was accepted for publication on August 17, 2012.

The authors have no funding, financial relationships, or conflicts of interest to disclose.

Send correspondence to Erin L. McKean, MD, Department of Otolaryngology, University of Michigan Health System, 1904 Taubman Center, 1500 East Medical Center Drive, Ann Arbor, MI 48109. E-mail: elmk@med.umich.edu

DOI: 10.1002/lary.23746 cranial fossa (Fig. 1). Endoscopic nasal biopsy of this lesion revealed sinonasal undifferentiated carcinoma (SNUC).

The patient was subsequently referred to our institution where surgical extirpation was performed. The anterior subcranial approach used at our institution for open skull base procedures has been previously described. ${ }^{1}$ The pathologic diagnosis of the surgical specimen confirmed SNUC (Fig. 2). Postoperatively, the patient underwent adjuvant cisplatin therapy and concurrent radiation therapy to a total dose of $48 \mathrm{~Gy}$.

One year after surgery, two intracranial lesions were detected on surveillance imaging. These lesions were dural-based and near the right pterion, suspicious for subarachnoid dissemination of the SNUC. The patient underwent radiosurgery, delivering 18 Gy to both lesions. One year following radiosurgery, MRI showed progression of an enhancing dural mass near the right pterion (Fig. 3). There was no evidence of additional metastatic disease or recurrence at the primary site. Neurosurgical extirpation of this enlarging mass was performed. Final pathology of this lesion confirmed high-grade nonintestinal adenocarcinoma (Fig. 4).

\section{Patient 2}

A 58-year-old man presented with an intranasal mass and underwent endoscopic biopsy of the lesion, which revealed esthesioneuroblastoma (Fig. 5). He underwent endoscopic subtotal sinonasal debulking and adjuvant radiation therapy to a total dose of 60 Gy to the primary site. Eight years later, he developed recurrent epistaxis, and a polypoid mass was identified in the right nasal cavity. $\mathrm{He}$ was subsequently referred to our institution for management of a suspected locally recurrent esthesioneuroblastoma. 


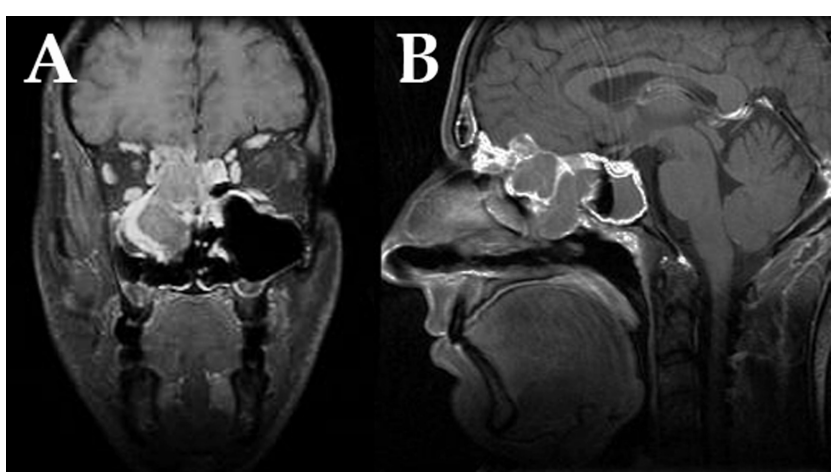

Fig. 1. Coronal $(A)$ and sagittal $(B)$ post-contrast T1-weighted MRI showing a mass centered in the right posterior ethmoid penetrating the cribriform plate into the anterior cranial fossa.

Imaging showed an enhancing mass centered in the left anterior and posterior ethmoid sinuses (Fig. 6). Endoscopic biopsy revealed intermediate-grade nonintestinal adenocarcinoma. Surgical extirpation was performed via an anterior subcranial approach. Pathologic review of the surgical specimen confirmed intermediate-grade nonintestinal type adenocarcinoma; chromogranin staining and
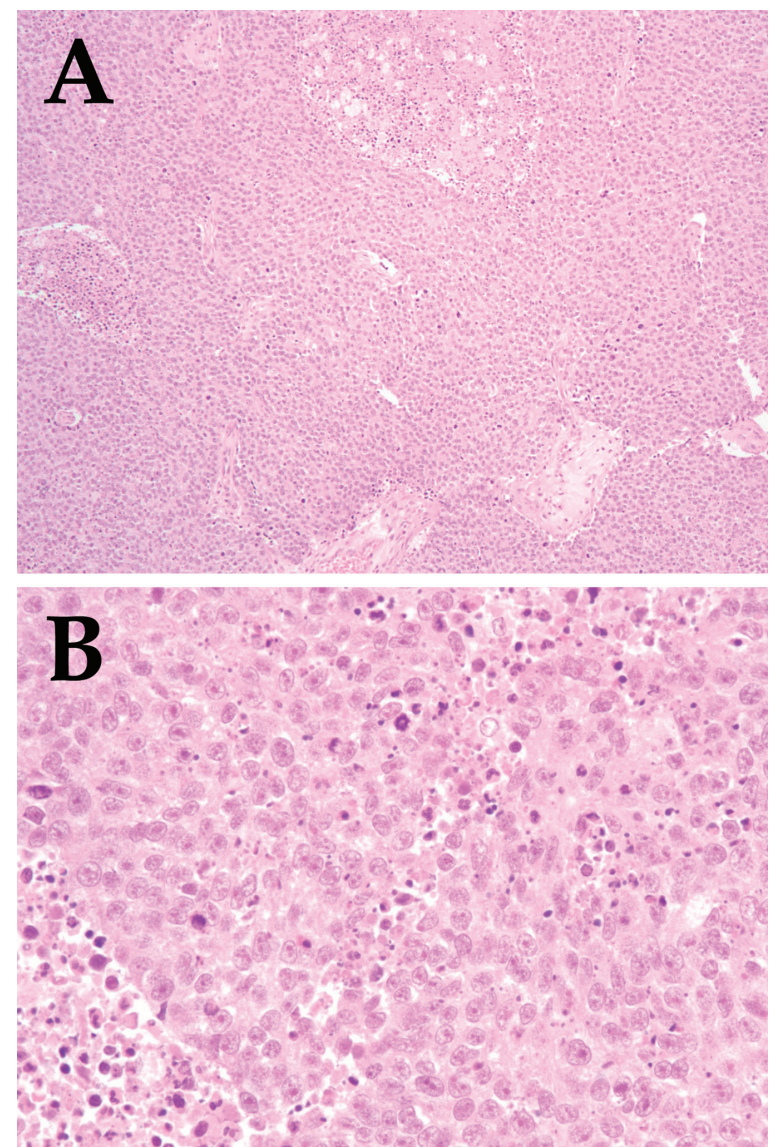

Fig. 2. Histopathologic features of high-grade SNUC in patient 1, displaying sheets of malignant cells with round to oval vesicular nuclei and frequent mitoses $(A)$ and no evidence of squamous, glandular, or neuroendocrine differentiation (B). Hematoxylin and eosin (H\&E) stain; original magnification $\times 10(A)$ and $\times 20(B)$.

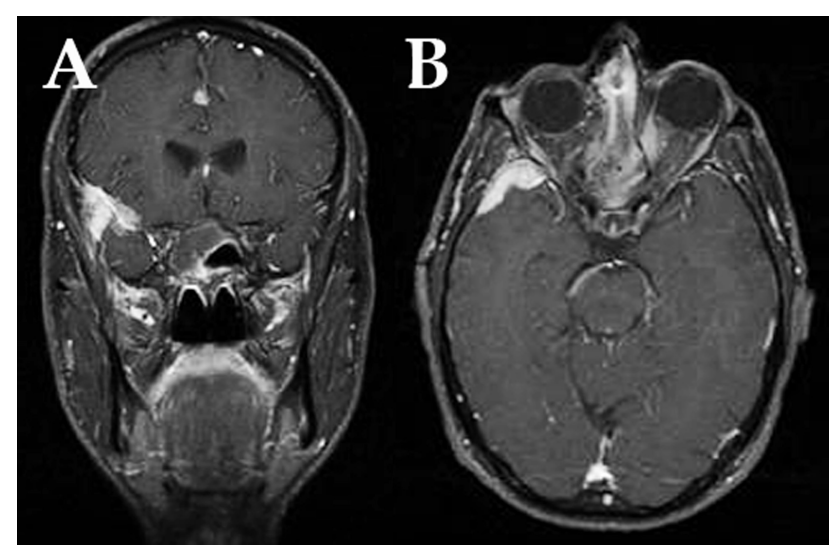

Fig. 3. Coronal (A) and axial (B) post-contrast T1-weighted MRI showing a persistent dural-based lesion in patient 1 one year following radiosurgery. Neurosurgical extirpation revealed nonintestinal adenocarcinoma.

pan-cytokeratin immunostaining suggest a change in tumor phenotype (Fig. 7).

\section{DISCUSSION}

Malignancies of the extra-axial anterior skull base represent a diverse group of histopathologic tumor
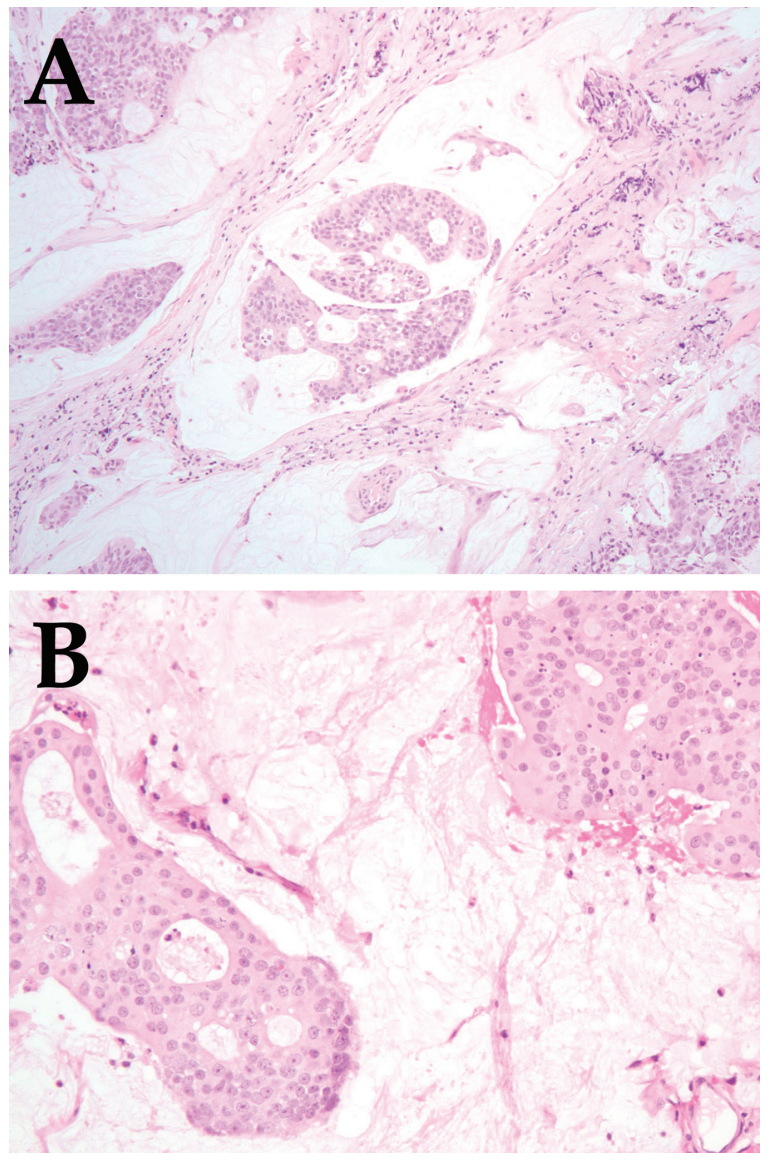

Fig. 4. Histopathologic features of adenocarcinoma in patient 1 , displaying mucinous material with scattered areas of gland-forming epithelium. H\&E; original magnification $\times 10(A)$ and $\times 20(B)$. 


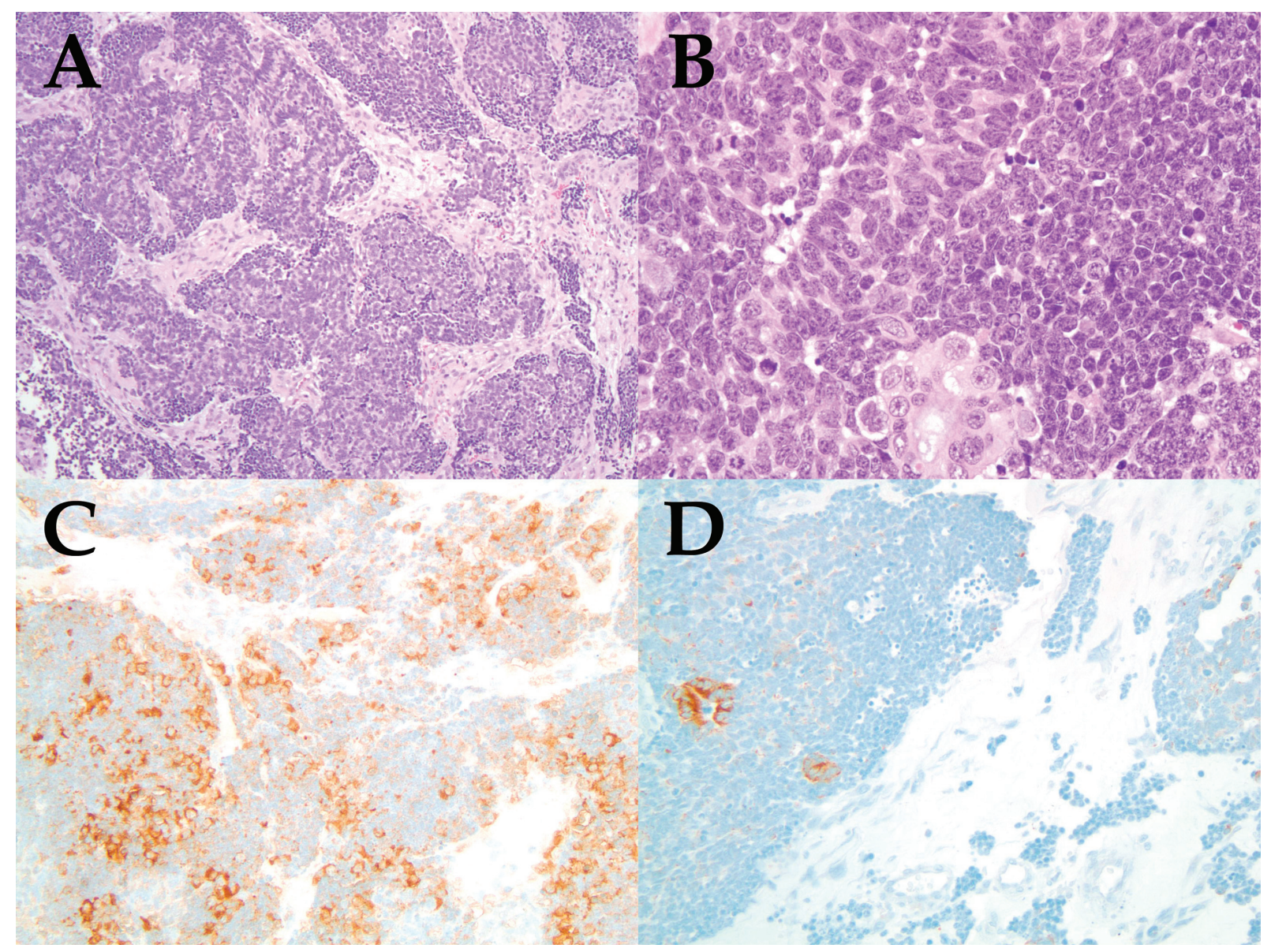

Fig. 5. Histopathologic features of esthesioneuroblastoma in patient 2, displaying nests of small round blue cells in the characteristic "salt and pepper" appearance, surrounded by vascular stroma (A and B). H\&E; original magnification $\times 10$ (A) and $\times 20$ (B). Chromogranin staining confirms a neuroendocrine tumor consistent with esthesioneuroblastoma (C) and pan-cytokeratin immunostaining, a marker for epithelial differentiation, corresponds with rare areas of glandular differentiation, but the majority of the tumor is negative (D). Original magnification $\times 10(C)$ and $\times 20(D)$.

pathology. This group includes, but is not limited to: squamous cell carcinoma, adenocarcinoma, adenoid cystic carcinoma, melanoma, esthesioneuroblastoma, SNUC, nasopharyngeal undifferentiated carcinoma, sarcoma, and skin malignancies. ${ }^{2-4}$ The management of

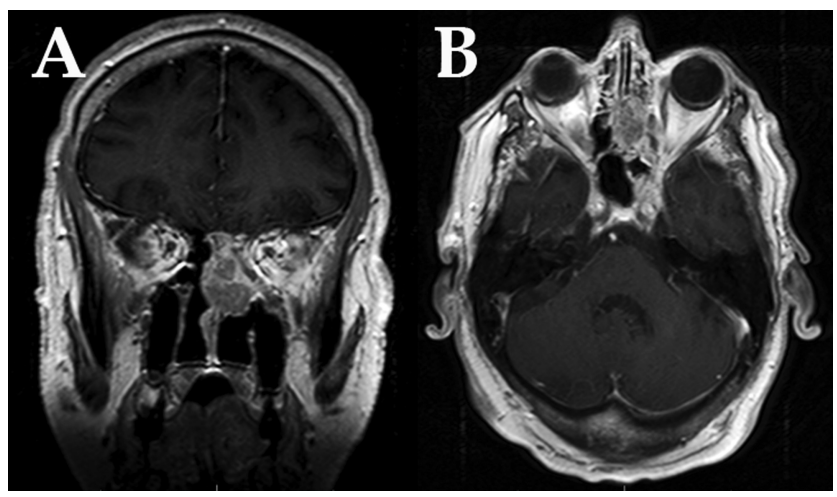

Fig. 6. Coronal (A) and axial (B) post-contrast T1-weighted MRI in patient 2. Surgical extirpation revealed nonintestinal adenocarcinoma. anterior skull base malignancies is dependent on a number of important factors. Accurate tumor histopathology is the cornerstone on which the treatment plan is formed, and also an important prognostic factor for predicting the likelihood of treatment success and overall survival in skull base malignancies. ${ }^{5,6}$ Likewise, the management of recurrent disease depends on the known biology of the primary tumor. This report describes unexpected findings in two patients with anterior skull base malignancies, presenting with suspected regional recurrence in patient 1 and suspected local recurrence in patient 2 , where the recurrent tumor encountered was of a distinct histopathologic type.

Sinonasal undifferentiated carcinoma was the original pathologic diagnosis in case 1. During this patient's initial resection, there was evidence of dural invasion; thus, the dural-based lesion that was found on postoperative tumor surveillance MRI was attributed to leptomeningeal spread of the SNUC. Review of the SNUC and nonintestinal adenocarcinoma specimens by a single pathologist (J.M.) confirmed both pathologic diagnoses. A second primary adenocarcinoma with dural metastasis is possible, although a second primary lesion 

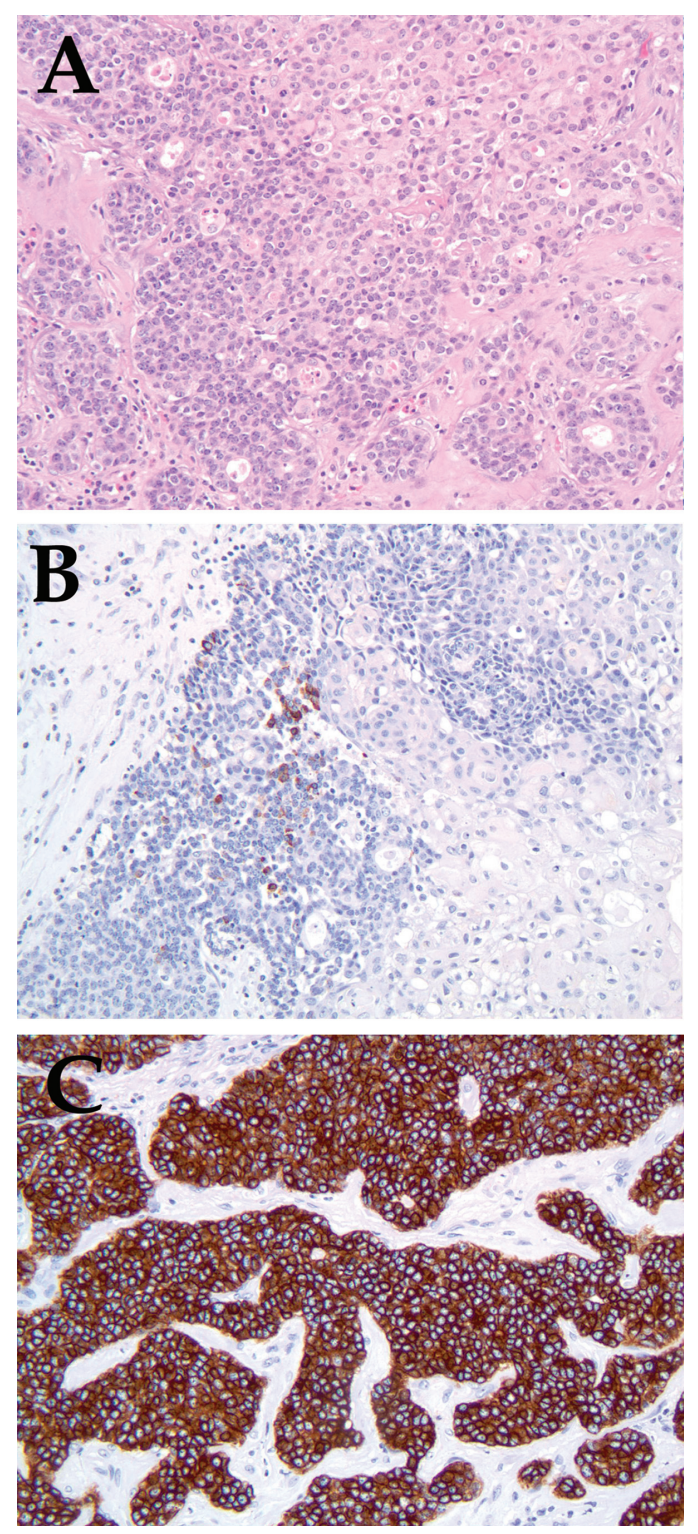

Fig. 7. Histopathologic features of adenocarcinoma in patient 2, displaying high-grade carcinoma with extensive gland formation (A). H\&E, original magnification $\times 15$. In contrast with Figure 5 , chromogranin staining of this tumor shows rare foci of uptake (B) and pan-cytokeratin immunostaining is strongly positive (C), signifying a change in tumor phenotype.

has yet to be identified in this patient in over 13 years of follow-up. Another possibility is the presence of a hybrid tumor consisting of multiple pathologic features, resulting in sampling error on the initial biopsy and surgical specimen. The initial tumor may have exhibited glandular pathologic features that were not identified due to sampling error during specimen processing. Finally, a rare genetic event resulting in transformation of the tumor from SNUC to adenocarcinoma may have occurred.

Esthesioneuroblastoma was the original pathologic diagnosis in case 2 . The patient presented with an intranasal mass and a suspected local recurrence. Review of the esthesioneuroblastoma and the nonintestinal adenocarcinoma specimens by a single pathologist (J.M.) confirmed the pathologic diagnoses. Although pathologic criteria for esthesioneuroblastoma were met with the original specimen, rare areas of glandular differentiation existed (Fig. 5). While esthesioneuroblastoma is in general a radiosensitive tumor, it is possible that glandular elements of the tumor were resistant to radiotherapy and ultimately resulted in the recurrence of this tumor as a nonintestinal adenocarcinoma.

\section{CONCLUSION}

Accurate tumor histopathology is imperative in the management of anterior skull base malignancies. This report describes two rare occurrences in which the authors encountered an unexpected and distinct tumor type when managing suspected locoregional recurrence of anterior skull base malignancies. The initial management of locoregional recurrence is often based on the known biology of the primary tumor. Although rare, the possibility of encountering a distinct tumor type in the posttreatment surveillance period must be considered in the management of recurrent anterior cranial base malignancies.

\section{BIBLIOGRAPHY}

1. Ross DA, Marentette LJ, Moore CE, Switz KL. Craniofacial resection: decreased complication rate with a modified subcranial approach. Skull Base Surg 1999;9:95-100.

2. Bhattacharyya N. Cancer of the nasal cavity: survival and factors influencing prognosis. Arch Otolaryngol Head Neck Surg 2002;128:1079-1083.

3. Katz TS, Mendenhall WM, Morris CG, Amdur RJ, Hinerman RW, Villaret DB. Malignant tumors of the nasal cavity and paranasal sinuses. Head Neck 2002;24:821-829.

4. Ketcham AS, Van Buren JM. Tumors of the paranasal sinuses: a therapeutic challenge. Am J Surg 1985;150:406-413.

5. Bentz BG, Bilsky MH, Shah JP, Kraus D. Anterior skull base surgery for malignant tumors: a multivariate analysis of 27 years of experience. Head Neck 2003;25:515-520.

6. Shah JP, Kraus DH, Arbit E, Galicich JH, Strong EW. Craniofacial resection for tumors involving the anterior skull base. Otolaryngol Head Neck Surg 1992;106:387-393. 